\title{
Modeling and Simulation of Upper Brachial Plexus Injury
}

\author{
Marie André Destarac, Cecilia E. García Cena, Roque J. Saltarén Pazmiño, Mónica J. Reyes Urbina, \\ Javier López López, and Ricardo Espinoza Gómez
}

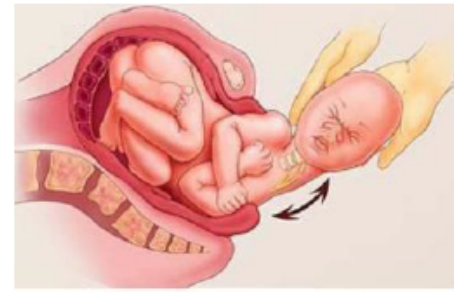

(a)

Fig. I. Brachial plexus paralysis during birth. (a) Shoulder dystocia. (b) Delivery in pelvic position [16]

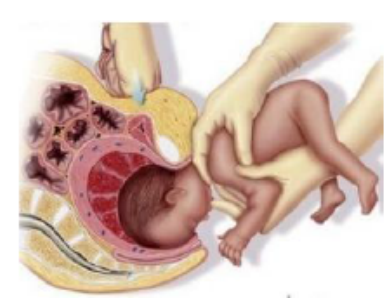

(b)

\section{INTRODUCTION}

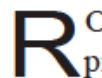
OBOTIC exoskeletons are an important tool for therapists. In recent years, several examples of their use can be found in the rehabilitation of upper limb injuries [1]-[4]. The advantage offered by robotic exoskeletons is the capacity to replicate with a patient the movements performed by a therapist during the treatment [4]. Many ofthem provide accurate data to monitor the recovery of the patient. In sorne cases, exoskeleton robots are used as an assistive device by the user to gain sorne degree of independen ce [6]-[8]. People with movement disabilities, such as brachial plexus injury, require assistive technology to feed themselves and perform other daily activities.

There are only a few examples of robotic exoskeletons developed specifically for the rehabilitation of patients with brachial plexus injury. One of them was designed by The Hugh MacMillian Rehabilitation Centre in Canada. The exoskeleton allows the patient to grasp objects with the hand using linear actuators for elbow and fingers ftexion [8]. Another example is Wilmington Robotic Exoskeleton, which is developed by $\mathrm{Al}$ duPont Hospital for Children [10]-[12].

The incidence of brachial plexus injury has not decreased over the last three decades. The estimated prevalence of brachial plexus injury during birth ranges from 0.2 to 2.0 per 1000 births, and significant arm weakness persists in 0.4 to 5.0 per 10000 births [13]-[15]. Obstetric brachial lesions present certain peculiarities: when produced at the moment of birth, they have a higher rate of recuperation than those seen in adult patients [2].
The mechanical forces acting on an infant during birth, particularly those of traction and compression, can produce a variety of lesions to the brachial plexus. For example, [12] discusses shoulder dystocia [this lesion is produced when the anterior region of the infant's shoulder is impacted on to the mothers pubic simphysis, as shown in Fig. 1(a)] during vaginal birth with a cephalic presentation. This may produce an over extension ofthe neck, causing a lesion to the plexus. Nevertheless, this type of lesion is also seen after a cesarean section and other vaginal births with an abnormal presentation; for example, a pelvic position shown in Fig. 1(b)].

This also [13] explains that risk factors may be maternal, fetal, or situations associated with a difficult birth. Maternalrelated risks highlighted include excessive weight, maternal diabetes, uterine abnormalities, and a history of brachial plexus paralysis in previous births. The only infant-related risk mentioned is a higher birth weight. Finally, birth-related risks include shoulder dystocia, prolonged labor, pelvic position, and the use of forceps or vacuum extractors during assisted delivery. 




(a)

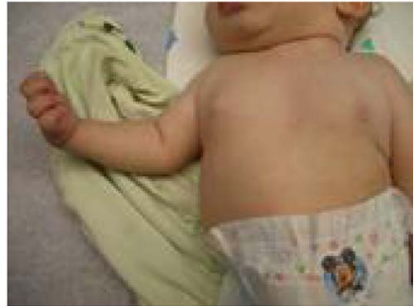

(b)
Fig. 2. Brachial Plexus Injuries. (a) Upper limb injury. (b) Lower limb injury [17].

The location of the lesion is determined through a clinical analysis; however, the type of lesion is established in correlation with the recovery time. For example, it is considered that if the superior roots are stretched or torn, an Erb palsy would occur, which is better known as "bad shoulder-good hand." In this type of palsy, there is a shoulder function deficiency and limited hand function, as shown in Fig. 2(a). In this lesion, the muscle groups affected are the external rotators, the shoulder abductors, elbow flexors, forearm supinators, and, frequently, the wrist extensors. In other words, the superior musculature is affected. On the other hand, if the inferior nerve roots are stretch or tear, a Klumpke palsy would occur. This palsy is known as "good shoulder-bad hand," a lesion wherein the muscles affected are the wrist flexors, finger flexors, and muscle intrinsic to the hand, as shown on Fig. 2(b). In a complete lesion, both the shoulder movements, as well as the hand movement, would be affected. In this type of lesion, all the roots within the plexus are involved [13].

The superior paralysis is the most frequent. It is estimated to represent almost $80 \%$ of the cases; more than $90 \%$ of this lesion resolves within a year of the infant's life. A complete paralysis appears in $20 \%$, and the inferior lesion has the lowest incidence $(2 \%)$.

According to [13], studies show that during the systematic exploration of the brachial plexus after a lesion only $20 \%-30 \%$ showed functional sequels, indicating an elevated rate of recovery. Classification of lesions may help to determine the long-term recovery prognosis. Early lesion detection and classification is crucial during the lactation period; otherwise, significant sequels over motor and sensitive functions may develop.

The treatment for brachial plexus injury starts in the neonatal unit, where a professional specialized in passive exercises initiates therapy and gives support to the family. It is particularly important to reduce the stress over the plexus both from the forces of gravity and traction. The prescribed physiotherapy starts mainly with passive and active isometric eccentric exercises. The exercise will reduce the risk of atrophy and muscle contractures in the long term. The persistence of symptoms beyond the first month of life suggests that the lesion could require specialized treatment, including surgical procedures that consist in a meticulous exploration of the plexus to determine the location and extension of the lesion. The lesion may require nerve graft, and the recovery time after surgery is usually between 7 and 9 months. After surgery, rehabilitation and nerve reeducation therapy would be necessary to improve strength sensitivity and movement of the affected extremity [13]. An exoskeleton can be used after the surgery with the intent to make the rehabilitation process more intense and controlled.

The main contributions of this paper are the following.

1) The presentation of the first musculoskeletal model that was developed in order to study the unilateral upper brachial plexus injury, which can be easily adapted to different anthropometric measures and degrees of affectation of the injury. This is imperative for this work because it is focused in children under 10 years, which is a target group that is still in a growth development stage.

2) The presentation of a new hardware and software architecture that allows an easy linking between the data capture of the arm movement of a patient with upper brachial plexus injury and the animation of the developed model. This way, it is possible to get accurate ranges and speeds of movement, which save and study the data. With the analysis of these results, it can obtain a more precise and reliable evolution and prognosis of the patient.

3) The simulation of the force that a patient with upper brachial plexus injury can develop in certain muscles while performing rehabilitation exercises and their comparison with the case of a healthy subject. The data force will help to calibrate the exoskeleton control system to adapt it to the patient's needs during the different stages of rehabilitation.

The information presented here, as well as the models described in Section II, provide the basis to properly design a musculoskeletal model to develop simulations. These simulations give relevant information for the design of a rehabilitation exoskeleton with the objective of supporting postsurgical treatment in a patient with upper brachial plexus injury.

The present model corresponds to a European adult male, since all the tests were done with a subject with these characteristics, but it can be easily modified for a child.

This work is part of a project for the Rehabilitation Section of the Infanta Sofia Hospital, Madrid, Spain.

\section{Methodology}

To develop a model of the upper brachial plexus injury, two well-known methods have been used in this paper. For the skeletal system, consisting of rigid links and joints, the Denavit-Hartenberg method was used. This necessitated a careful study of human shoulder anatomy and the selection of the degrees of freedom (DoF) associated with the lesion. For the muscle-tendon system, the Hill-Zajac model was used, and only those muscles involved in the injury were selected. Fig. 3 shows the scheme of the methodologies used to create the musculoskeletal model.

\section{A. Musculoskeletal Modeling of Upper Brachial Plexus Injury}

The human shoulder is an extremely sophisticated and interrelated system that can produce a wide variety of complex 




Fig. 3. Scheme of the two methodologies used to create the musculoskeletal model.

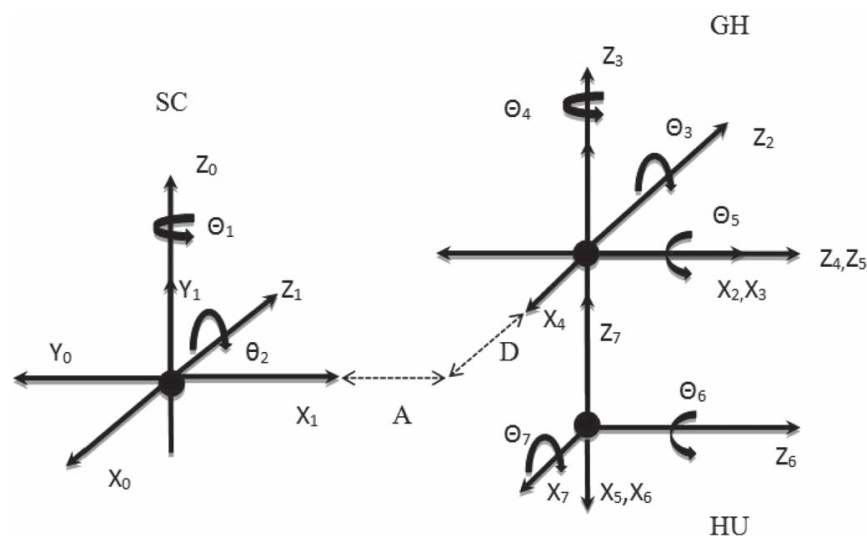

Fig. 4. Reference system for a 7-DoF shoulder girdle representation according to D-H convention.

movements [18]. In [19], it is stated that, given the complexity of the human shoulder, it is convenient to model the shoulder girdle (clavicle and scapula) as a closed kinematic chain, whereas the glenohumeral (GH) joint should be modeled as a serial chain. In [20], the shoulder girdle is modeled as an element with 5 DoF: $3^{\circ}$ for the $\mathrm{GH}$ joint and $2^{\circ}$ for the sternoclavicular (SC) joint.

Based on the aforementioned, the elements used in this paper to simulate the movement of the shoulder are the SC and GH joints and collarbone as one rigid link. The coordinate systems of each joint, proposed by the authors of this paper and previously published [21], are using the Denavit-Hartenberg convention and are shown in Fig. 4. As can be seen, each reference system is associated with the kinematic chain described before: $2 \mathrm{DoF}$ for the $\mathrm{SC}$ joint and $3 \mathrm{DoF}$ for the $\mathrm{GH}$ joint. Furthermore, in this paper, $2 \mathrm{DoF}$ have been added to represent the elbow joint, corresponding to flexion/extension and pronation/supination of the forearm. These are also presented in Fig. 4 and correspond to the humeroulnar (HU) joint.

It is worth noting that there are two offsets with respect to the axes modeled. 1) Longitudinal displacement along the $x_{1}$-axis (distance $\mathrm{A}$ in Fig. 4). This corresponds to the distance between the SC joint and the GH joint region (clavicle length).

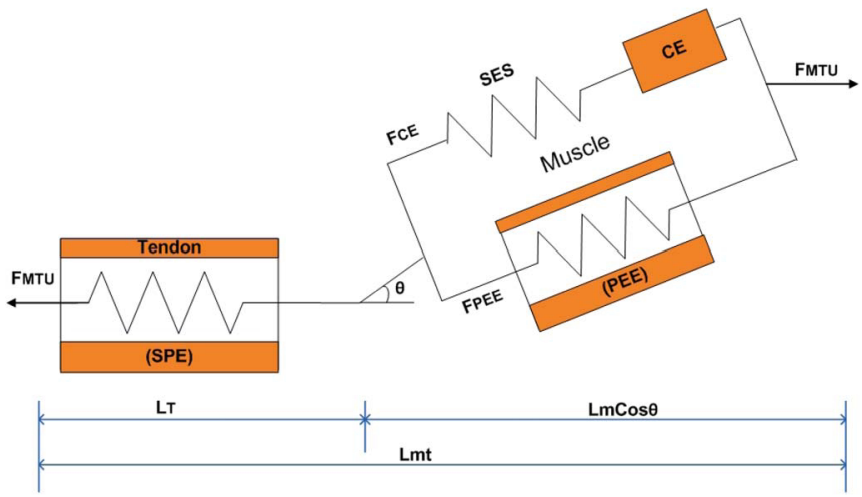

Fig. 5. Hill-Zajac muscular force model. FMTU: Force of MTU; SPE: Series Passive Element; FCE: Force of CE; FPEE: Force of Parallel Elastic Element; SES: Series Elastic Element; PEE: Parallel Elastic Element; CE: Contractile Element; $\theta$ : pennation angle.

2) Displacement along the $z_{2}$-axis, which represents the distance between the GH joint and scapula (distance D in Fig. 4). The values considered in this paper are $13 \mathrm{~cm}$ for constant $\mathrm{A}$ and $7.3 \mathrm{~cm}$ for constant $\mathrm{D}$.

We explored the particular case of an upper brachial plexus injury in postsurgery state, in which the patient begins rehabilitation with negligible mobility in the shoulder, due to limited capacity of innervated muscles. In this case, only the GH and elbow joints functions need to be considered, making it possible to carry out the basic tasks of the rehabilitation process.

Most computational models that have been proposed to simulate the mechanical behavior of muscles are based on the mathematical model of Hill [22], which considers the muscular system as a single-input-single-output model. The input parameter corresponds to the neuronal signal, whereas the force is the output parameter. The mechanical behavior of a muscle-tendon unit (MTU) can be represented in a simplified 1-D model, since the force and displacement occur mainly in the direction of muscle fibers [23].

The model consists of three components, and each one is associated with a specific muscle characteristic. The contractile element (CE) represents the contraction process that generates the active force, the series elastic element (SES) represents the internal elasticity of the muscle cell, and the parallel elastic element (PEE) is responsible for maintaining muscle structure (stiffness) with passive loads and opposes elongation.

To complete the model of Hill, Zajac [24] incorporates tendon elasticity through a series passive element (SPE), as shown in Fig. 5. This is true, because the muscle is attached to the bone through the tendon. The resistance of the MTU will depend on the stiffness and length of the tendon.

Muscle tissue was studied both in active and passive states [25]. The passive force ( $\left.F_{\text {Passive }}\right)$ component corresponds to the resistance of muscle tissue before deformation and is given primarily by the connective tissue. The active force $\left(F_{\text {Active }}\right)$ component is produced by the interaction of the proteins that cause muscle contraction [26].

$F_{\mathrm{MTU}}$ can be deduced from Fig. 5, corresponding to the force of the MTU which is defined as

$$
F_{\mathrm{MTU}}=F_{\mathrm{PEE}}+F_{\mathrm{CE}}=\left(F_{\text {Passive }}+F_{\text {Active }}\right) \cos \theta .
$$


The force produced by CE is transmitted to SPE and corresponds to the active force. PEE exerts an independent force corresponding to the passive force. The total force in the muscle is the sum of the active and passive components, which are transmitted with the same magnitude through the tendon to produce muscle movement. Some studies have characterized these forces [27]-[29].

The displacements of the CE are independent of the SES. The sum of both displacements is equal to the displacement of PEE, which corresponds to the total length of muscle $\left(L_{M}\right)$. The total length of the MTU is the sum of the length of PEE and SPE

$$
L_{M T}=L_{T}+L_{M} \cos \theta .
$$

The passive force is the resistance of the muscle to be compressed or extended and is the result of the viscous and elastic properties of the muscle and the elastic properties of the tendon. This is defined by the following equation:

$$
F_{\text {Passive }}=f_{p l 1}+f_{\alpha} * f_{p l 2}
$$

where $f_{p l 1}$ and $f_{p l 2}$ represent the viscoelasticity and elastic properties of the muscle fiber, respectively. $f_{\alpha}$ represents the force-excitation signal relationship, and it is also present in active force. This relationship describes the dependence of the active force generated by the muscle and the excitation signal received.

The active force developed by the muscle depends on several factors such as length, muscle contraction rate, duration time of contraction, and muscular architecture. There is an optimum length to which the muscle can generate a maximum force known as Maximal Isometric Force $\left(F_{0}\right)$. The active force can be expressed as a function of a number of dimensionless parameters [24], except $F_{0}$, thus affecting the magnitude

$$
F_{\text {Active }}=F_{0} * f_{l} * f_{\text {vel }} * f_{\alpha}
$$

where $f_{l}$ represents the force-length relationship. Force or tension produced by the muscle is not constant and varies as it changes its length. $f_{\text {vel }}$ represents the force-velocity relationship and can be interpreted as the greater the load applied to the muscle, the lower the speed at which the muscle can contract.

The number of factors that affect $F_{\text {Active }}$ can be increased depending on the complexity of the numerical model to be developed. For purposes of this study, three major relationships presented in (4) are acceptable.

The mathematical model previously presented applies to the muscles of a healthy subject, during daily activities such as personal cleanliness, eating, grasping objects, etc. In the case of subjects with upper brachial plexus injury, the affected muscles are paralyzed, being only able to produce passive forces and unable to produce active forces [30]. The affected muscles are deltoid, supraspinatus, infraspinatus, teres minor, biceps brachii, brachioradialis, brachialis, and supinator [31].

Each muscle has particular architectural parameters that are required for a functional musculoskeletal model. In addition to the muscles aforementioned, we have included in
TABLE I

Muscle ARChitecture IMPLEMENTED IN THE ModeL

\begin{tabular}{|l|c|c|c|c|}
\hline \multicolumn{1}{|c|}{ Muscle } & $\begin{array}{c}\text { Mass } \\
\text { (g) }\end{array}$ & $\begin{array}{c}\text { Optimal } \\
\text { fascicle } \\
\text { length (cm) }\end{array}$ & $\begin{array}{c}\text { Optimal } \\
\text { tendon length } \\
\text { (cm) }\end{array}$ & $\begin{array}{c}\text { Maximal muscle- } \\
\text { tendon length } \\
\text { (cm) }\end{array}$ \\
\hline Short Biceps & 161.4 & 14 & 13.5 & 34 \\
\hline Long Biceps & 170 & 11 & 17 & 34 \\
\hline Brachialis & 284 & 10 & 3 & 16 \\
\hline Brachioradialis & 112 & 19 & 6 & 31 \\
\hline Pronator Quad & 25 & 2.4 & 2.2 & 5 \\
\hline Pronator Teres & 80 & 7.9 & 5 & 13 \\
\hline Supinator & 58 & 3.7 & 2 & 6.5 \\
\hline Long Triceps & 290 & 18 & 11 & 31 \\
\hline Medial Triceps & 204 & 10 & 0.3 & 12 \\
\hline Lateral Triceps & 518 & 14 & 10 & 25 \\
\hline Deltoid clavicular & 124 & 14.7 & 2 & 21.2 \\
\hline Deltoid acromial & 377 & 6.7 & 8.6 & 18 \\
\hline Deltoid scapular & 300 & 17 & 5.9 & 24 \\
\hline Supraspinatus & 90 & 9.2 & 4.6 & 15.6 \\
\hline Infraspinatus & 226 & 6.8 & 5.6 & 15.3 \\
\hline Teres minor & 39 & 5.7 & 4.6 & 12.6 \\
\hline Teres major & 232 & 14 & 5.8 & 21 \\
\hline
\end{tabular}

the model others relevant to perform different movements for rehabilitation. The muscle architecture parameters for an adult male are taken from [32] and [33]. Table I summarizes the values used.

Section I explains the treatment performed in order to try to correct the brachial plexus injury. It explained that in cases where it is recommended to perform a surgical procedure for reconstructing the brachial plexus, nerve grafts are placed. Grafts can be partial or total, in case there is a need to completely remove the brachial plexus. Depending on this, certain muscles that were paralyzed will recover their ability to act. As a result, rehabilitation becomes a valuable tool for the patient, seeking to restore the muscle contraction capacity to generate force.

In [34] and [35], several experiments were carried out with newly innervated muscles following brachial plexus reconstruction. These subjects showed $20 \%$ contraction capacity, as compared with a healthy muscle. Rehabilitation aims to gradually increase this percentage.

In the literature on arm rehabilitation [28], [30], [35], two of the basic movements are elbow flexion-extension and arm abduction-adduction, which are vital for important activities such as feeding or personal cleanliness. Furthermore, in reviewing the diagnostic and therapeutic protocols in hospitals such as Infanta Sofía in Madrid, Spain, the authors corroborated that these movements are two of the most explored in rehabilitation therapies of upper brachial plexus injury.

For this reason, the following section provides details of the musculoskeletal model created to simulate these movements using real data from a motion sensor placed on the arm and forearm of a healthy male test subject.

\section{B. Hardware and Software Architecture for Model Animations}

This section presents the software and hardware architecture developed to perform kinematics animations and dynamic simulations of the musculoskeletal model. 


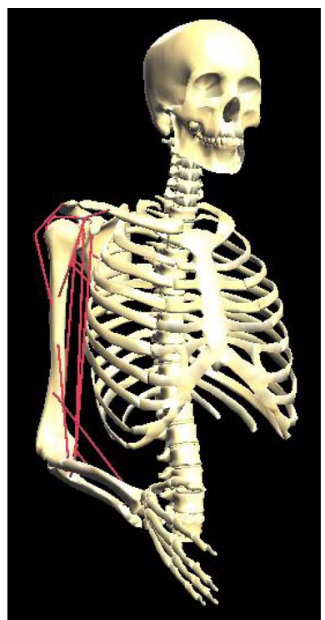

Fig. 6. Musculoskeletal model of upper extremity.

The software used to create the musculoskeletal model of the upper limb is the Musculoskeletal Modeling Software (MSMS), developed by the University of Southern California [36]. The muscle model that is used by this software is proposed by Hill-Zajac, and in [37], it shows the structure of the muscle model implemented in MSMS.

This software offers the possibility to perform kinematics animations of previously recorded data motion and also to perform also simulations with data generated from Simulink. The main difference between them is that the animations require data from the evolution in time of the angles that represent a movement (first pathway of Fig. 7), whereas the simulation requires the activation of one or more muscles of the model to determine its dynamic evolution and other parameters such as the force exerted by the muscles due to the activation signal (second pathway of Fig. 7). During the simulation, it is also possible to see the model animation.

We use the 2.2 version of MSMS, which also contains some examples, including one of the human upper limbs. This example was modified to have the configuration required for this paper. Muscles shown in Table I were added to the model as line segment, and it configured the muscle architecture parameters for each one. Furthermore, wrapping objects were included that interact with line segments to obtain more realistic movements [38]. Fig. 6 shows the model created.

The active joints in this model are shoulder (with $3 \mathrm{DoF}$ ), elbow (with $2 \mathrm{DoF}$ ), and wrist (with $2 \mathrm{DoF}$ ). Each joint range of motion has been restricted according to the values provided by Kapandji [39]. The movement of the scapula and clavicle has been disabled to maintain the stability of the model [40].

In order to know the position of the patient's arm while performing particular movement, Razor 9 DOF AHRS inertial motion unit (IMU) of Sparkfun Electronics was used. The calibration and data processing of the IMU was presented in [21]. In that work, the IMU data were merged by the Direction Cosine Matrix to give in response Euler angles; these are stored in a text file. It also presented a versatile and userfriendly interface, making it possible to graph and obtain the numerical values of the evolution of the Euler angles in real time and offline.

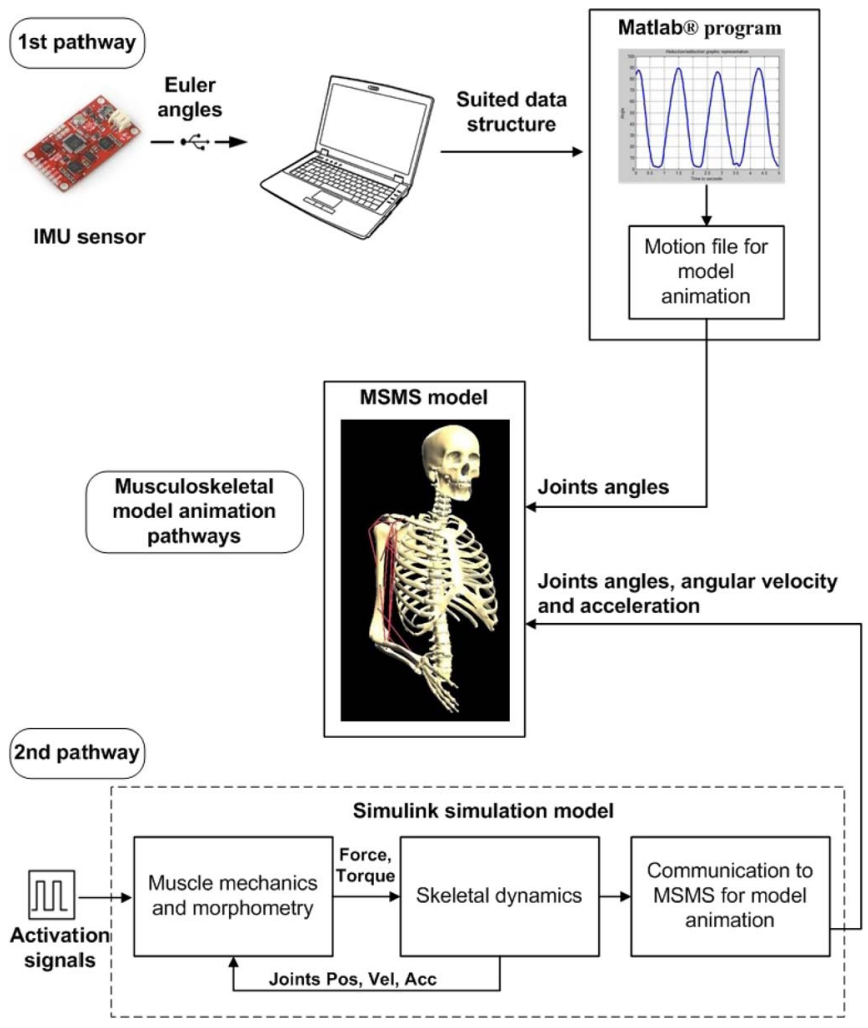

Fig. 7. Hardware and software architecture implemented for musculoskeletal model animation.

\section{Procedure for Obtaining Results}

As mentioned before, with the musculoskeletal model, it is possible to perform a kinematics animation of a movement. IMU was used to obtain the data that represents the movement and then the data was sent to MSMS. In order to do that, a program in MATLAB was created to receive Euler angles data from the IMU via the USB port. In addition, data was prepared to carry out a movement animation and saved in a file. In other words, the data from the IMU is suited to be consistent with the reference system model created in MSMS. Fig. 7 shows how the data from IMU is sent to MSMS to perform the movement animations (see 1st pathway). MSMS uses a special file to perform the animations, known as Motion File Format, and contains data in ASCII format.

As aforementioned, we explored movements of the rehabilitation therapy, such as elbow flexion-extension and arm abduction-adduction. For the animation of both movements, an IMU was placed for each case, as shown in Fig. 8.

According to [39], elbow flexion/extension movement has a range between $0^{\circ}$ and $150^{\circ}$, where $0^{\circ}$ represents that the arm is fully extended and $150^{\circ}$ represents total elbow flexion (with the arm close to the body, allowing only the movement of the forearm); thus, the angular variation graph must show values within this range. The arm abduction/adduction movement has a range between $0^{\circ}$ and $180^{\circ}$, where $0^{\circ}$ represents that the arm is fully extended and $180^{\circ}$ represents total arm abduction. In most of the cases, patients with upper brachial plexus injury are unable to cover the full range of this movement, so that the animation was made for the half of the range, i.e., from $0^{\circ}$ to $90^{\circ}$. 


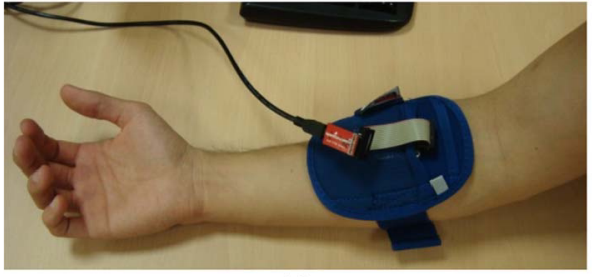

(a)

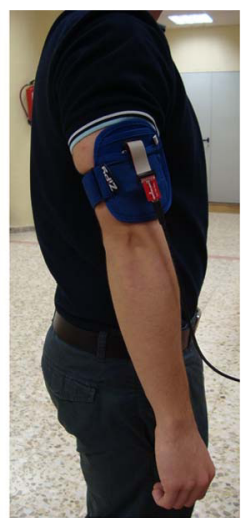

(b)
Fig. 8. Position of the IMU for animation. (a) Elbow flexion/extension. (b) Arm abduction/adduction.

MSMS has a simulation tool to convert the musculoskeletal model in a Simulink blocks diagram. One of the blocks has as input parameters the activation signals of each muscle of the model and will generate (as output) a determined force to move the segment to which they are attached. Both programs are able to communicate with each other through the User Datagram Protocol (UDP). Thus, it is possible to obtain model motion animation from Simulink data, which are sent over UDP to the MSMS. The kinematic response (animation) that is obtained with the activation signals can be seen directly on the model, as shown in Fig. 7 (second pathway).

After converting the model to its representation in Simulink, we proceeded to activate three muscles to study their behavior and its effect on the dynamics of the musculoskeletal model. We selected the brachialis, brachioradialis, and biceps brachii (short and long), which develop the main function of elbow flexion and extension. The brachial muscle contributes $47 \%$ of the maximum torque required to perform elbow flexion and extension, biceps $34 \%$ and brachioradialis 19\% [41].

A pulse train was used to activate them, simulating the electrical stimulation technique or functional electrical stimulation (FES) [37]. For the three muscles, a pulse train with amplitude of 1 and a period of $2 \mathrm{~s}$, pulsewidth of $50 \%$, and active delay of $1 \mathrm{~s}$, was selected. It is important to note that MSMS restricts the range of the excitation signal from 0 to 1 , and this range represents the percentage of muscle activation. This means that brachialis, biceps, and brachioradialis muscles are active at $100 \%$ and would be the case of a healthy subject.

\section{RESULTS}

\section{A. Model Animations}

Figs. 9 and 10 are the resulting graphs obtained with the IMU data. According to the arm biodynamic characteristics [42], it was expected that the resulting signals had a sinusoidal shape and a low frequency, because movements were made slowly.

The result was correct, because the model performs the movement of the forearm while carrying out the elbow flexion and extension. Fig. 11 shows three sequences of the animation of this movement.

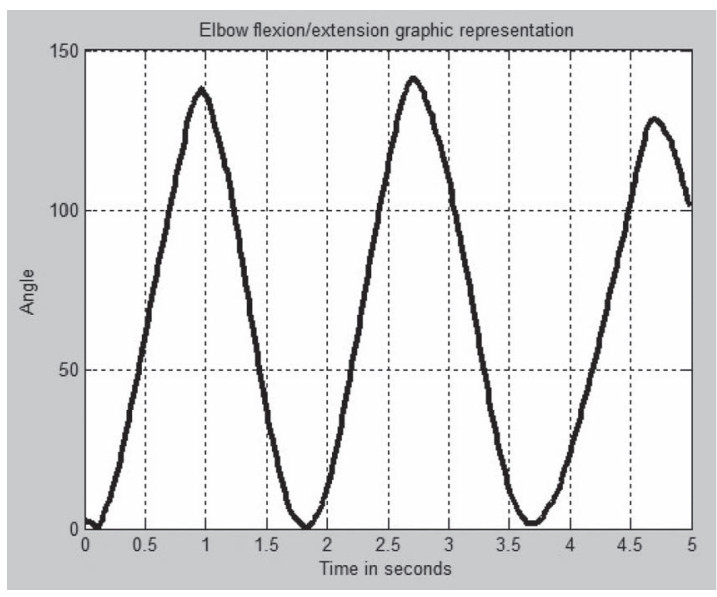

Fig. 9. Elbow flexion-extension motion representative graph.

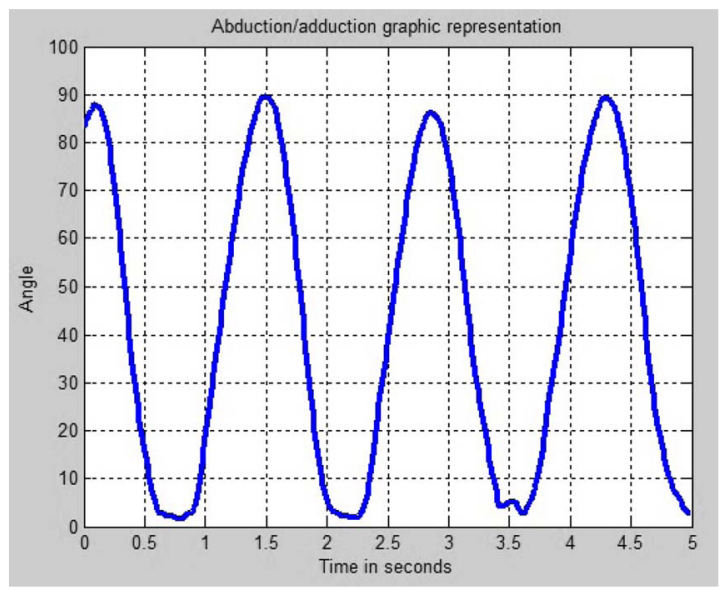

Fig. 10. Arm abduction/adduction motion representative graph.

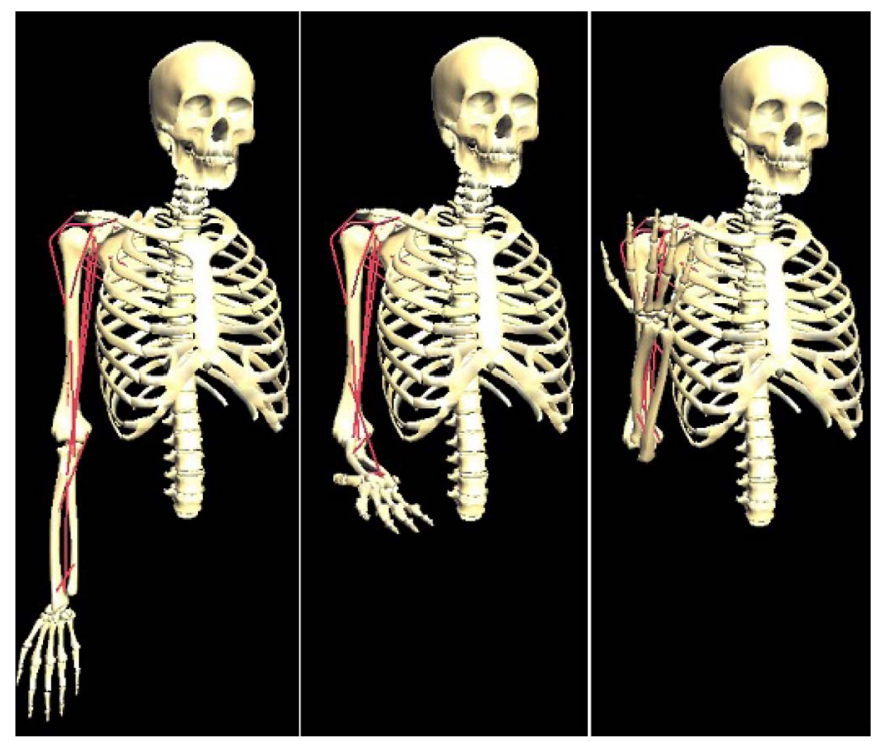

Fig. 11. Animation sequence of elbow flexion-extension.

There were also other movement animations related to the rehabilitation of brachial plexus injury, and in all cases the musculoskeletal model correctly performed the animation of each one. With these results, one can proceed with the motions simulation in order to obtain muscle force data. 


\section{B. Simulations of Muscle Force}

Fig. 12 shows the response of the muscles, which corresponds to the force developed due to the activation signal. The results obtained are consistent with those reported in [43]. In the simulation of the movement, it was possible to see that the forearm muscles lift achieved elbow flexion and extension in full range of motion.

In order to simulate the case of a patient with upper brachial plexus injury in an immediate postoperative state, the activation signals of brachialis and biceps brachii muscles were modified, reducing its amplitude to $20 \%$, i.e., 0.2 . Brachioradialis muscle was activated again with the same amplitude previously used. The force response is shown in Fig. 13. In this case, the activated muscles were not able to do the elbow flexion and extension in full range of motion.

The same steps previously described were performed for the simulation of abduction-adduction of the arm. The muscles that perform this movement are the deltoid and supraspinatus. They were activated at $100 \%$ with the same pulse train showed in Fig. 12. The forces obtained are shown in Fig. 14 and are consistent with the results reported in [44]. Fig. 15 shows the results for both muscles when they were activated at $20 \%$.

\section{DISCUSSION}

The MSMS selected has a feature that was important for this paper, which consists in the ability to set specific parameters of muscle and bone architecture, allowing more reliable simulations. This is of most importance when studying the range of motion and force that a person with upper brachial plexus injury can perform. In addition, it was preferred that the selected software could provide data related to the dynamics of the model, such as a force and torque. These become critical aspects to properly select engines for rehabilitation exoskeleton. These data, among others, are provided by MSMS.

The model presented in this paper accurately reproduced the movements made during the tests using the data provided by the IMU. The kinematic animations were very helpful to analyze the behavior of the muscles, whereas these perform the movements that are being studied and to correct the musculoskeletal model when force or angle data were meaningless due to errors at the point of origin or insertion of the muscles.

Through simulations, in which electrical pulses are sent to the muscles involved in elbow flexion-extension and arm abduction-adduction, it was possible to compare the difference of force production in healthy muscle in contrast to one that has been recently reinnervated, to repair an upper brachial plexus injury. For healthy muscles, the model shows that they produce enough force to lift the forearm (flexion-extension) and arm (abduction-adduction) in complete range of motion. In Figs. 12 and 14 , this event corresponds to the points where it reaches a greater magnitude of force.

In the simulation of the case of a patient with brachial plexus injury, the force produced by the muscles to make elbow flexion-extension is not sufficient to completely lift the forearm, which justifies the use of a rehabilitation device. Observing Figs. 12 and 13, the force difference produced in both cases is obvious. A dramatic decrease of the force generated


Fig. 12. Response of brachialis, biceps brachii, and brachioradialis muscles when they are active at $100 \%$ using the FES stimulation technique. 



Fig. 13. Response of muscles when brachialis and biceps brachii are active at $20 \%$ and brachioradialis at $100 \%$.


Fig. 14. Response of deltoid and supraspinatus muscles when they are active at $100 \%$ using the FES stimulation technique.



Fig. 15. Response of muscles when deltoid and supraspinatus are active at $20 \%$. 
by the brachialis and biceps brachii muscles can be noted. The decrease in amplitude of the activation signal also reduces the force produced. This affects the response of the brachioradialis muscle, which is forced to increase its force dueto the dynamic imposed by the model.

The same applies to the case of the arm abduction-adduction. It is shown in Fig. 15 that the force generated by activating the muscles of $20 \%$ is negligible, and it is impossible to carry out the movement.

\section{CONCLUSION AND FUTURE WORK}

The study and analysis in this work on aspects such as the up- per brachial plexus injury, mathematical modeling of the mus- eles, and musculoskeletal modeling have been the foundation necessary to develop exoskeletons for the rehabilitation of patients with this lesion. The design of rehabilitation exoskeletons must take into account various factors such as responsiveness of muscle, force produced by them, and joint torque required.

Although the developed musculoskeletal model is not com- plete, since it does not include all the muscles involved in the movements of the upper limb, it is a first prototype that allows different movements, including two relevant to the re- habilitation of the brachial plexus. It also helps to reveal the dynamic and kinematic consequences produced by an affected or atrophied muscle.

Future work includes the improvement of the musculoskeletal model, adding the missing muscles related to the movement of the shoulder. The model will be adapted to the anthropo-metric measures and muscle architecture of a child less than 10 years old. It is also expected to collect data from patients suffering this type of injury, extending the validation of this musculoskeletal model.

Real-time data collected by the IMU will also be linked to the model, so that the movements performed by the human subject can be immediately reproduced in the musculoskeletal model. This can be integrated into a virtual environment, in which the patient tries to reach a given virtual object as a therapy exercise.

\section{ACKNOWLEDGMENT}

The authors would like to thank H. Spratt, D. Bamforth, and L. Bamforth for their contribution in the revision of the English language of this work. The authors also thank J. M. Sánchez for his help during the tests with the IMU. 